\title{
Imaging Nanomedicine-Based Drug Delivery: a Review of Clinical Studies
}

\author{
Francis Man, ${ }^{1}$ Twan Lammers, ${ }^{2,3,4}$ Rafael T. M. de Rosales ${ }^{1}{ }^{1}$ \\ ${ }^{1}$ School of Biomedical Engineering \& Imaging Sciences, King's College London, St Thomas' Hospital, Westminster Bridge Road, London, \\ SE1 $7 E H, U K$ \\ ${ }^{2}$ Department of Nanomedicine and Theranostics, Institute for Experimental Molecular Imaging (ExMI), University Clinic and Helmholtz \\ Institute for Biomedical Engineering, RWTH Aachen University, Pauwelsstrasse 30, 52074, Aachen, Germany \\ ${ }^{3}$ Department of Targeted Therapeutics, MIRA Institute for Biomedical Technology and Technical Medicine, University of Twente, \\ P.O. Box 2177500 AE, Enschede, The Netherlands \\ ${ }^{4}$ Department of Pharmaceutics, Utrecht Institute for Pharmaceutical Sciences (UIPS), Utrecht University, Universiteitsweg 99,3584 CG, \\ Utrecht, The Netherlands
}

\begin{abstract}
Imaging plays a key role in the preclinical evaluation of nanomedicine-based drug delivery systems and it has provided important insights into their mechanism of action and therapeutic effect. Its role in supporting the clinical development of nanomedicine products, however, has been less explored. In this review, we summarize clinical studies in which imaging has provided valuable information on the pharmacokinetics, biodistribution, and target site accumulation of nanomedicine-based drug delivery systems. Importantly, these studies provide convincing evidence on the uptake of nanomedicines in tumors, confirming that the enhanced permeability and retention (EPR) effect is a real phenomenon in patients, albeit with fairly high levels of interand intraindividual variability. It is gradually becoming clear that imaging is critically important to help address this high heterogeneity. In support of this notion, a decent correlation between nanomedicine uptake in tumors and antitumor efficacy has recently been obtained in two independent studies in patients, exemplifying that image-guided drug delivery can help to pave the way towards individualized and improved nanomedicine therapies.
\end{abstract}

Key words: Nanomedicine, Drug delivery, Liposome, PET, SPECT, MRI, Ultrasound, Nanoparticle, Companion diagnostic, EPR

\section{Introduction}

Drug delivery systems based on nanoparticle technologies have been explored for more than 40 years, being one of the most active multidisciplinary fields of research to date [1]. Of the several drug delivery platforms available, liposomes, polymers, and solid inorganic nanoparticles have been the most widely studied. The rationale behind these drug carrier systems is to exploit their specific pharmacokinetic and biodistribution properties to deliver sufficient therapeutic

Correspondence to: Rafael T. M. de Rosales; e-mail: rafael.torres@kcl.ac.uk amounts of the drug cargo to the specific target(s) (the drug being usually a toxic and/or insoluble small molecule), and to reduce its side effects due to local controlled release. To date, several nanomedicine drug delivery systems based on these concepts have been translated into clinical products, these include Abraxane ${ }^{\circledR}$, DaunoXome ${ }^{\circledR}$, Doxil ${ }^{\circledR} /$ Caelyx $^{\circledR}$, Marqibo ${ }^{\circ}$, Myocet ${ }^{\circledR}$, and Onivyde ${ }^{\circledR}$ among others, with more in clinical trials ( $>45$ found in ClinicalTrials.gov).

Imaging has played a very important role in the progress of this field. From a developmental perspective, it allows the non-invasive measurement of the biodistribution and pharmacokinetics of these drug delivery systems in animal 
models of disease, allowing us to select the best candidates by providing answers to important questions such as "Where do they go inside the body?," "How long do they stay?," "How are they cleared?," "Are they reaching the target? and if so, how much?," and "Is the drug being released?" Several imaging techniques are available to obtain such information. To gain a deeper understanding of the different preclinical imaging modalities available - with their intrinsic advantages and disadvantages - and how they have supported the preclinical development of drug delivery systems - we refer the reader to several recent reviews on this area [2-7].

In the clinical setting, imaging can play an additional role due to human and disease heterogeneity. It is widely accepted that human/disease heterogeneity affects the efficacy of all therapies and is particularly detrimental to the clinical effectiveness and translation of therapeutic nanomedicines [8-10]. Unlike in animal models of disease, where most frequently the same genetic strains of mice and disease cell lines are used to assess the efficacy of drug delivery systems, in humans, heterogeneity is present between patients with different diseases, those with the same disease, and even within different lesions of the same patient. This heterogeneity has led to nanomedicines being approved based on their improved safety profile compared to conventional drugs, rather than improvements in therapeutic efficacy [11]. Hence, to overcome this problem, imaging methods that allow us to predict at the patient-to-patient level the efficacy of drug delivery systems, as well other therapeutics, could play important roles in the future [9].

The objective of this review is to analyze the progress and future prospects in the area of imaging drug delivery in humans. Our aim is twofold: first, we want to provide a descriptive analysis to date on how different clinical imaging techniques have played an important role in providing proofof-concept data to support the development of drug delivery systems into clinical products. Second, we aim at highlighting how recent studies are shining new light into the interand intra-patient heterogeneity problem and how imaging can be used to predict drug delivery/therapeutic efficacy. Where possible, we have also tried to highlight findings from all of these studies that, in our opinion, deserve further attention.

To identify clinical studies in the field of image-guided drug delivery, we searched PubMed in January 2018, using combinations of the following terms: drug delivery, imaging, liposome, MRI, nanomedicine, nanoparticle, PET, radiolabeled, scintigraphy, SPECT, and ultrasound. Results were then restricted to clinical trials and articles were manually screened for relevance (Table 1). Studies in which imaging, most frequently x-ray computed tomography (CT) and 2-deoxy-2- $\left[{ }^{18} \mathrm{~F}\right]$ fluoro-D-glucose (FDG) positron emission tomography (PET), was used solely for monitoring response to treatment were not included. With the focus being on nanomedicine drug delivery systems, studies of labeled small molecules and antibodies were not considered.
For a brief overview of antibody-based theranostics, we refer the reader to the excellent review by Moek et al. [32]. The results of our search showed that liposome technologies are the most studied drug delivery system in humans. For reviews on image-guided drug delivery focused more specifically on clinical applications of liposomes in combination with imaging, we refer the reader to recently published reviews by Petersen et al. [33] and Lamichhane et al. [34]. Our search results also highlighted that the majority of the clinical studies of imaging-guided drug delivery to date have been performed using nuclear imaging modalities, principally planar gamma scintigraphy with only a minority using single-photon emission computed tomography (SPECT) or PET. This was followed by fewer studies using ultrasound (US) and magnetic resonance imaging (MRI). A brief description of each imaging technique is provided in each section, and the reader will find an excellent overview and comparison of the various imaging techniques in the introduction to molecular imaging by James and Gambhir [35]. All results have been organized in two levels, imaging technique and disease.

\section{Clinical Studies Using Gamma Scintigraphy and Single-Photon Emission Computed Tomography Imaging}

Gamma scintigraphy and single-photon emission computed tomography (SPECT) imaging rely on gamma-emitting radioisotopes, most commonly technetium- $99 \mathrm{~m}\left({ }^{99 \mathrm{~m}} \mathrm{Tc}\right.$, $\left.t_{1 / 2}=6.0 \mathrm{~h}, \gamma=140 \mathrm{keV}\right)$, indium-111 $\left({ }^{111} \mathrm{In}\right.$, $t_{1 / 2}=2.8$ day, $\left.\gamma=171 \mathrm{keV}, 245 \mathrm{keV}\right)$, iodine-123 ( ${ }^{123} \mathrm{I}$, $\left.t_{1 / 2}=13.2 \mathrm{~h}, \gamma=159 \mathrm{keV}\right)$, or iodine-131 $\left({ }^{131} \mathrm{I}\right.$, $t_{1 / 2}=8.0$ day, $\gamma=364 \mathrm{keV}$ ) in the clinic. The signal is captured by a gamma camera, equipped with collimators to localize the origin of the signal. Conventional scintigraphy provides two-dimensional (2D) images. In SPECT imaging, the gamma camera is rotated around the patient to obtain multiple $2 \mathrm{D}$ projections which can then be reconstructed into a three-dimensional (3D) image. Current clinical SPECT scanners provide a spatial resolution of $8-10 \mathrm{~mm}$, a temporal resolution of a few minutes and a sensitivity of $10^{-10}$ to $10^{-11} \mathrm{~mol} / \mathrm{l}$ of radiotracer [35].

\section{Oncology}

Most of the studies involved imaging liposomes radiolabeled with Tc-99m or In-111 to evaluate drug delivery to tumor sites. Following preclinical studies showing accumulation of liposomes in tumors, early clinical studies were aimed at establishing both the safety of the liposomes and their use as imaging agents for tumor detection and staging [36-39]. For example, Lopez-Berestein et al. [12] administered Tc-99mlabeled liposomes to seven cancer patients. It was unclear at the time whether early-generation liposomes selectively 


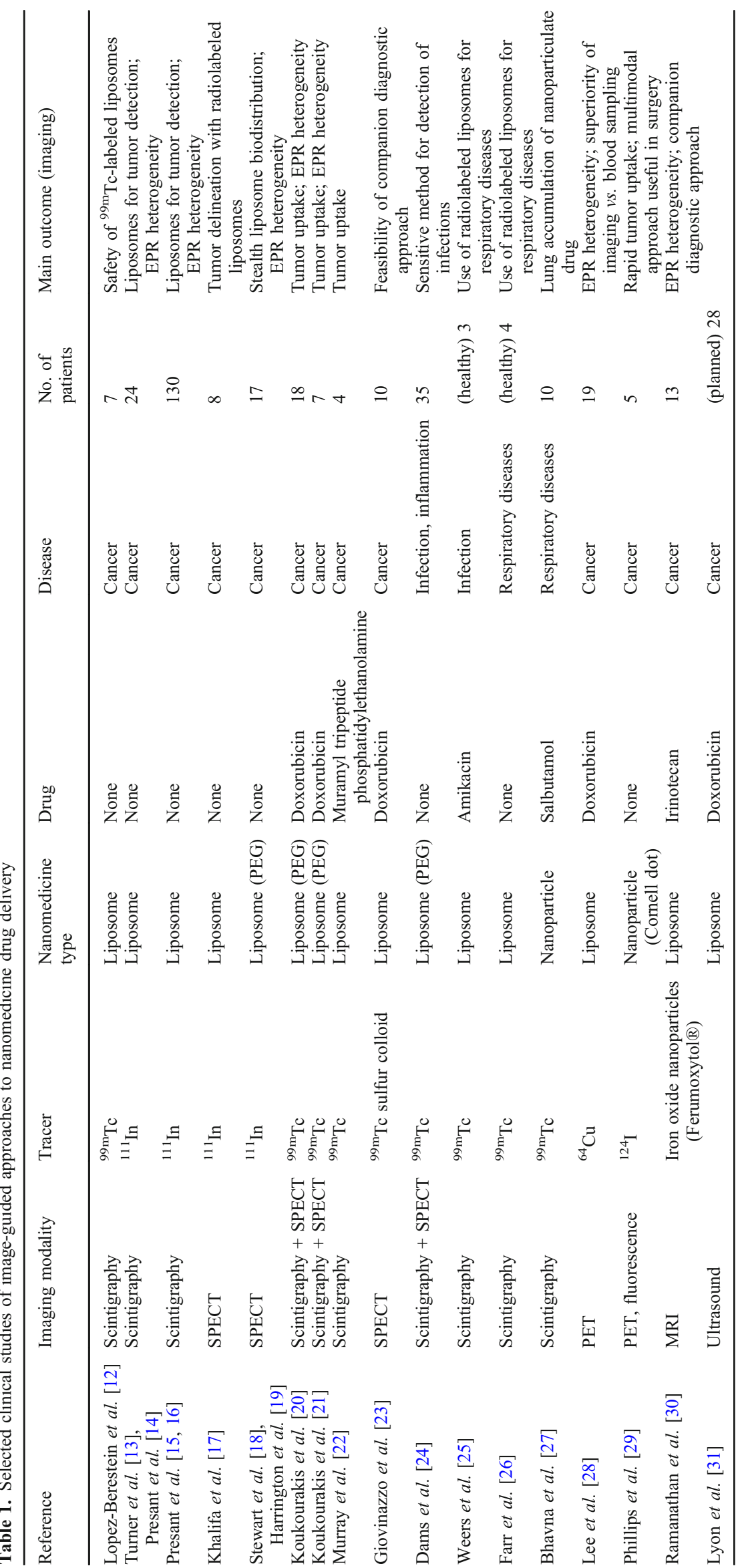


accumulated at tumor sites, and therefore liposomes were primarily seen as a means to reduce the toxicity of their payload or to target macrophages. The study revealed the accumulation of liposomes in macrophage-rich tissues such as the lungs, liver, and spleen, but did not mention any accumulation at the tumor sites. It is, however, unlikely that this could have been observed, considering that the four out of seven patients had various forms of leukemia, and one of those with a solid tumor was in complete remission. The value of this study lies more in its demonstration of the safety and relative ease of using radiolabeled liposomes in humans. The safety of the technique was confirmed in the first clinical study using In-111-labeled liposomes, conducted by Turner et al. [13, 14]. Although the study was unblinded, accumulation of liposomes was observed at known tumor sites in 22 out of 24 patients, and revealed unsuspected tumors in 3 patients, demonstrating the utility of radiolabeled liposomes for tumor detection. Furthermore, a high variability of liposomal uptake in tumors was noted, possibly the first observation of enhanced permeability and retention (EPR) effect heterogeneity in humans. The same group later published what remains by far the largest clinical trial of radiolabeled nanomedicines, in terms of number of patients imaged [15]. Even on this larger scale, In-111labeled liposomes were safe to use, and the authors reported 70-80\% sensitivity and $90 \%$ specificity for non-cerebral tumor detection. Scintigraphic images of patients with Kaposi's sarcoma and head and neck cancer (HNC) showed accumulation of radioactivity at the tumor sites [16]. The authors concluded to the usefulness of In-111-labeled liposomes as a diagnostic tool and of liposomes in general as drug delivery vehicles. Later, Khalifa et al. reported low uptake but excellent tumor delineation in seven out of eight patients with high-grade glioma using In-111-labeled liposomes [17].

Two particularly noteworthy studies are those performed by Stewart, Harrington, and colleagues [18, 19]. By then, liposome technology had witnessed the development and clinical approval of so-called stealth, i.e., polyethylene glycol (PEG)-coated, or PEGylated, liposomes with increased circulation times [40]. The first study aimed at establishing the biodistribution pattern of stealth liposomes in 17 patients with solid tumors. The extended circulation time compared to earlier-generation liposomes [41] was confirmed by scintigraphic imaging. Tumor accumulation of the radiolabeled liposomes was evident, in some cases for up to 10 days after administration, with eightfold variations in uptake between tumors (Table 2). In the second, more detailed study, 15 patients with locally advanced breast cancer, lung cancer, cervix cancer, or squamous cell HNC, or high-grade glioma, were administered In-111-labeled PEGylated liposomes, with the aim of obtaining precise information on the pharmacokinetics of these liposomes. One of the observations made was that of the daily urinary excretion of a small percentage of the injected In-111, presumably caused by the slow degradation of the liposomes in the tissues. This preliminary observation deserves further investigation. Indeed, beyond measuring liposomal uptake in the tumor, radiolabeling liposomes could provide a means of quantifying the amount of drug released from the liposomes. In the present case, In-111 urinary excretion might be used as a surrogate marker of drug release from the liposomal formulation. Scintigraphy showed a long circulation time of the liposomes and accumulation mostly in the liver and spleen. This was accompanied by a high stability of the liposomes that remained in circulation up to 4 days postadministration. Because this slow clearance from the blood led to high background signal, accumulation of the In-111-labeled liposomes in the tumors was not observed in the first $48 \mathrm{~h}$; however, the tumors were eventually visualized in 15 of the 17 patients studied. One of the main findings from this trial was that liposomal accumulation in the tumors could be seen for up to a week after administration. This was demonstrated in the striking scintigraphic image of a patient with Kaposi's sarcoma, illustrating the EPR effect in humans (Fig. 1).

The second lesson was a remarkably large variability of liposome uptake observed in the tumors, even after accounting for tumor size. In contrast, uptake in the main organs (liver, spleen, lungs, kidneys) was rather uniform between different patients. Although no data were available to explain this heterogeneity, variability in tumor vascularization and local inflammation were proposed as plausible explanations, based on preclinical and other clinical studies. The use of radiolabeled liposomes to predict drug uptake in patients, in other terms patient stratification, was proposed by the authors as a way to improve response rates in phase II clinical studies.

A common feature of the studies described above is that the liposomes contained no other cargo than the radiotracer. The first clinical studies of radiolabeled liposomes containing a therapeutic drug were published in 1999 and 2000 by Koukourakis et al. [20, 21]. These pilot studies aimed at evaluating the combination of liposomal doxorubicin $\left(\right.$ Caelyx $\left.{ }^{\circledR}\right)$ with radiotherapy. In the first study, nine patients with non-small cell lung cancer (NSCLC) and seven patients with $\mathrm{HNC}$ were administered Caelyx ${ }^{\circledR}$ radiolabeled with Tc$99 \mathrm{~m}$ and imaged by planar gamma scintigraphy. Accumulation of the liposomes in the tumor sites was observed $2 \mathrm{~h}$ after administration, with tumor-to-blood ratio increasing after $10 \mathrm{~h}$. In the NSCLC patients, liposomal uptake in the tumors correlated with the degree of tumor micro-vascularization, showing the presence of the EPR effect. The second study describes the same experiment in seven patients presenting locally advanced sarcomas [21] (Fig. 2). The apparent absence of liposomal drug-related toxicity and the high response rate (4 complete responses out of 7 patients) were considered encouraging, despite the low number of subjects and the absence of a control group. Although no measurement of doxorubicin levels by either imaging or biopsy was described, scintigraphy showed accumulation of Tc- $99 \mathrm{~m}$ at tumor sites previously determined by CT or bone scans, on average 2.8 -fold more than in neighboring normal 
Table 2.. EPR heterogeneity: variability of radiolabeled liposome uptake in tumors. Adapted with permission from the American Association for Cancer Research: Harrington et al. [19]

\begin{tabular}{|c|c|c|c|c|c|c|}
\hline Patient & Tumor & Stage & Whole body scan & SPECT & Total $\%$ injected dose ${ }^{a}$ & $\% \mathrm{ID} / \mathrm{kg}^{\mathrm{b}}$ \\
\hline 1 & SCC bronchus & T4N0M0 & Positive & Positive & 1.7 & 12.5 \\
\hline 2 & SCC bronchus & T4N0M0 & Positive & Positive & 1.6 & 25.4 \\
\hline 3 & Breast (ductal) & T4N2M1 & Negative & Negative & & \\
\hline 4 & SCCHN & T3N2M0 & Positive & Positive & 3.5 & 46.8 \\
\hline 5 & Breast (ductal) & T4N1M0 & Positive & Positive & 0.3 & 2.7 \\
\hline 6 & Breast (ductal) & T4N2M1 & Positive & Positive & 1.5 & 3.9 \\
\hline 7 & Breast (ductal) & T3N2M0 & Positive & Positive & 1.7 & 9.5 \\
\hline 8 & SCCHN & T4N0M0 & Positive & Positive & 0.7 & 24.2 \\
\hline 9 & $\mathrm{SCCHN}$ & $\mathrm{T} 3 \mathrm{~N} 1 \mathrm{M} 0$ & Positive & Positive & 1.0 & 32.0 \\
\hline 10 & SCC cervix & FIGO IIIB & Negative & Positive & NA & NA \\
\hline 11 & Breast (ductal) & T4N2M0 & Positive & Positive & 1.4 & 5.2 \\
\hline 12 & SCC bronchus & T2N0M1 & Negative & Negative & & \\
\hline 13 & SCCHN & T3N2M0 & Positive & Positive & 0.6 & 9.0 \\
\hline 14 & SCCHN & T3N0M0 & Positive & Positive & 1.6 & 53.0 \\
\hline 15 & SCC bronchus & T3N0M1 & Positive & Positive & 2.6 & 16.7 \\
\hline 16 & Glioma (AA) & Inoperable & Negative & Positive & NA & NA \\
\hline 17 & Glioma (GBM) & Inoperable & Negative & Positive & NA & NA \\
\hline
\end{tabular}

${ }^{\text {a }}$ Tumor uptake determined from ROI on 72-h whole-body scan

${ }^{\mathrm{b}}$ Percentage injected dose $/ \mathrm{kg}$ calculated from estimated tumor volume

$S C C$, squamous cell cancer; $A A$, anaplastic astrocytoma (grade III); $G B M$, glioblastoma multiforme (grade IV); $N A$, not assessable (tumor uptake was only measurable from whole-body scans)

tissue. This again demonstrates the increased uptake of liposomes in tumors in humans. Finally, another study described liposomal mifamurtide (Mepact $\left.{ }^{\circledR}\right)$ radiolabeled with Tc-99m and administered to four cancer patients for pharmacokinetic analysis, showing accumulation in lung metastases in two patients [22].
More recently, Giovinazzo et al. [23] investigated an indirect approach to imaging liposomal drug delivery, using Tc-99m sulfur colloid (TSC, Technecoll ${ }^{\circledR}$ ) to inform on the distribution of PEGylated liposomal doxorubicin (Doxil $\left.{ }^{\circledR}\right)$. TSC and other Tc-99m-labeled colloids are clinically approved and routinely used for

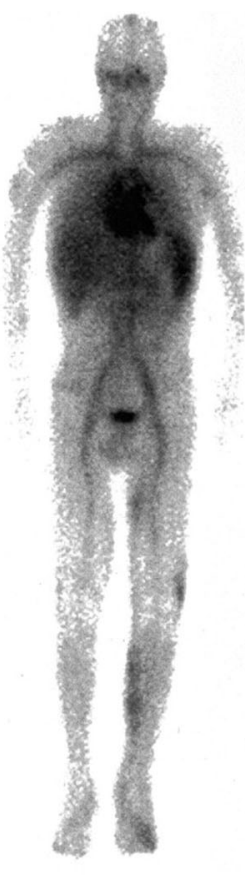

4 hours

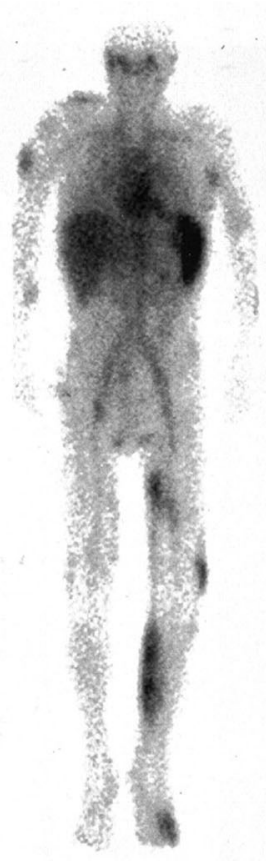

24 hours

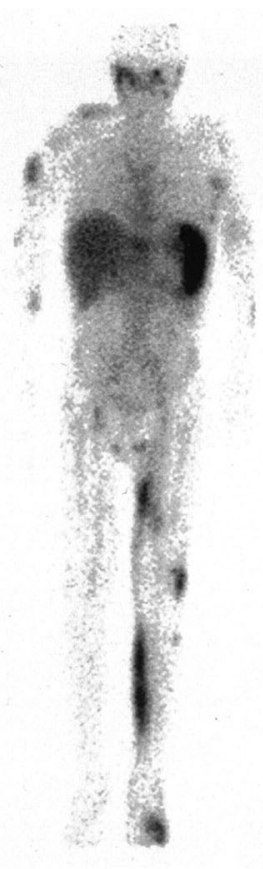

72 hours

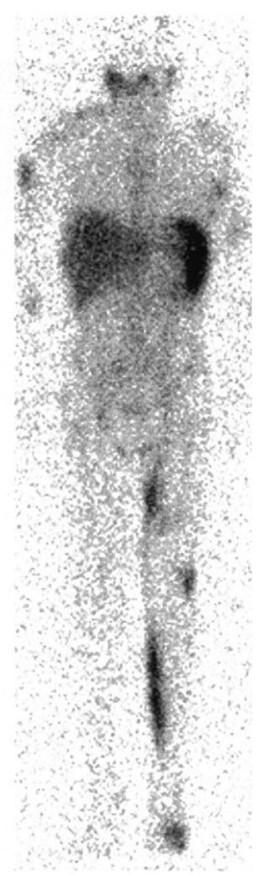

7 days

Fig. 1. Whole-body gamma camera images over 7 days of a patient with Kaposi's sarcoma administered In-111-labeled liposomes. Areas of liposome uptake in the left foot and leg, right arm, and face corresponded with typical Kaposi's sarcoma lesions. Prolonged retention of the radiolabel is seen despite significant clearance of circulating liposomes, as demonstrated by disappearance of the cardiac blood pool image. Adapted with permission from the American Association for Cancer Research: Harrington et al. [19]. 


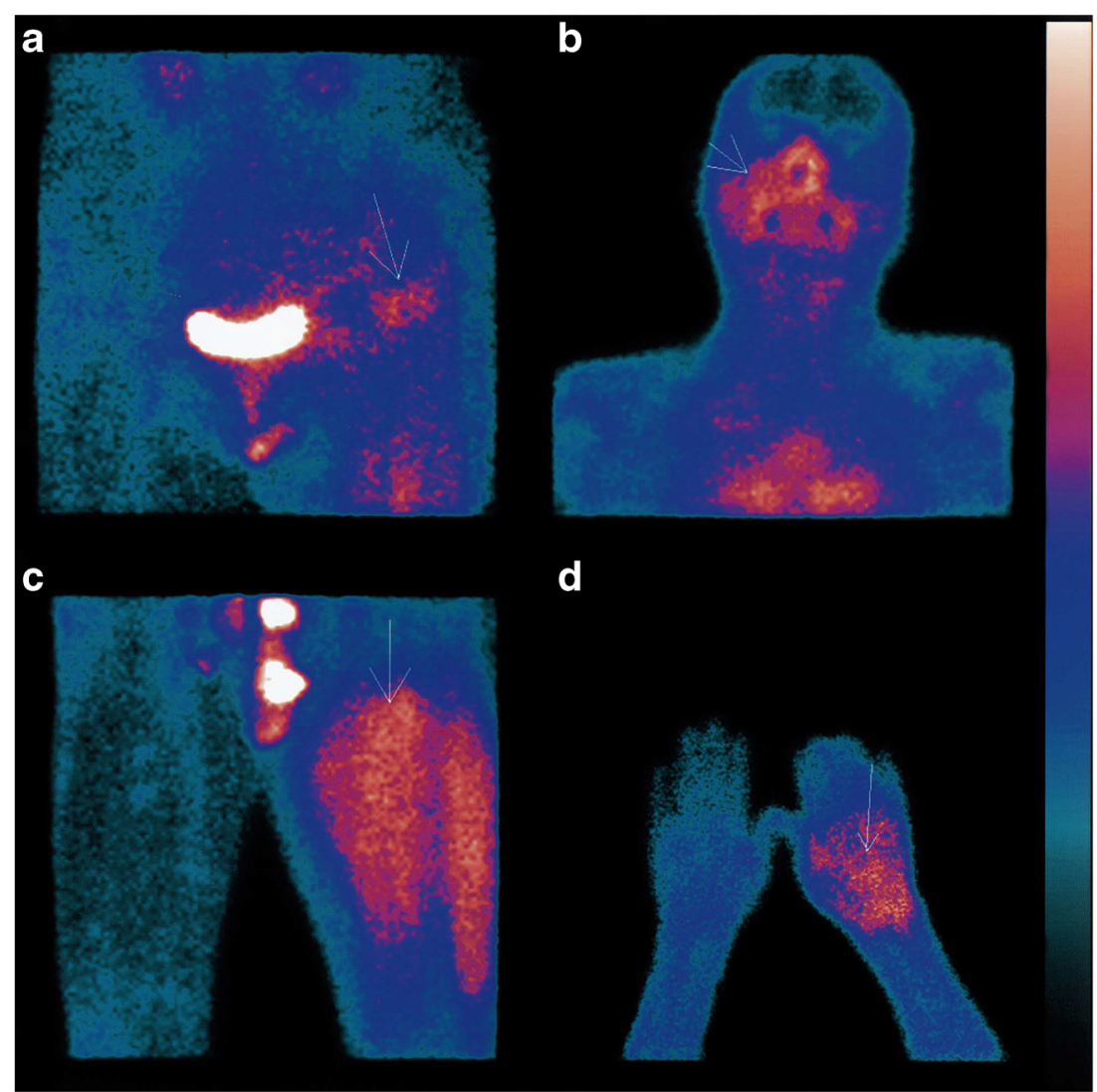

Fig. 2. Scintigraphic planar images from four patients with sarcoma administered ${ }^{99 m}$ Tc-labeled liposomal doxorubicin $($ Caelyx $($ ) $)$. a Clockwise from top left: fibrosarcoma of the iliac region, $\mathbf{b}$ angiosarcoma of the maxillary andrum, $\mathbf{c}$ Ewing sarcoma of the femur, d Kaposi sarcoma of the palmar area. Adapted with permission from Koukourakis et al. [21].

lymphoscintigraphy and staging of cancers [42]. Since the uptake of PEGylated liposomes is at least in part mediated by the mononuclear phagocyte system (MPS), the hypothesis was that the biodistribution of TSC, which is also cleared through the MPS, would mirror that of Doxil®. Thus, TSC uptake might be used to predict the uptake of Doxil ${ }^{\circledR}$ and thereby inform on the potential efficacy and/or toxicity of this drug, in particular the risk of developing palmar-plantar erythrodysesthesia (PPE). Ten patients with epithelial ovarian cancer were administered TSC, 1 week before commencing treatment with liposomal doxorubicin. Imaging of the spleen, liver, and hands was done by scintigraphy and SPECT/CT. Levels of TSC in the hands were highly variable from one patient to another and did not correlate with blood TSC levels, suggesting variability in MPS activity. After finding a positive correlation between blood levels of TSC and encapsulated doxorubicin, the authors derived a formula to estimate doxorubicin levels in the hands based on TSC measures only. This estimated value positively correlated with clinical grades of PPE severity. The main advantage of this indirect approach is that it is based on a clinically approved product and could potentially be used as a general predictor of uptake for nanomedicines that are cleared through the MPS. On the other hand, it would still require initial correlation studies to be undertaken for each nanomedicine, whereas radiolabeling of the therapeutic nanomedicine directly and specifically informs on the uptake of the drug.

\section{Infection and Inflammation}

In an interesting departure from cancer studies, Dams et al. [24] evaluated the use of ${ }^{99 \mathrm{~m}} \mathrm{Tc}$-labeled PEGylated liposomes for the detection of infection and inflammation, as an alternative to the clinical standards represented by radiolabeled immunoglobulin $\mathrm{G}(\mathrm{IgG})$ and white blood cells. Here, each patient acted as their own control, being first administered the liposomes and $24 \mathrm{~h}$ later ${ }^{111} \mathrm{In}$-labeled $\mathrm{IgG}$. Several interesting findings arose from this study. From a diagnosis perspective, there was excellent concordance between the results from the scans with Tc-99m-labeled liposomes and In-111-labeled IgG, with discordance in only 1 out of 35 patients. The calculated specificity was identical for both methods, and the sensitivity was higher with the liposomes. The liposomes also allowed better delineation of the foci of accumulation than the IgG in some patients, presumably 
because of the lower rate of reverse diffusion of the liposomes into the blood pool. It should be noted that the suspected foci were predominantly musculoskeletal and the radiolabeled liposomes failed to detect the single case of endocarditis in the study, highlighting a limitation of this approach. As expected, the PEGylated liposomes showed a long circulation time, with signal in the blood pool still visible $24 \mathrm{~h}$ after administration. Although the concordance study required the use of a shorter-lived isotope for the first scan to allow a second scan as quickly as possible, one might consider the use of a longer-lived isotope in future studies. This would allow scanning the patient later, potentially improving the signal-to-background ratio and thereby facilitating diagnosis. Later scans may also allow monitoring the response to treatment. Alternatively, modifications of the physico-chemical properties of the liposomes might allow faster clearance from the circulation for more rapid increases in target-to-background ratios, resulting in earlier diagnosis. The authors considered radiolabeled liposomes as a diagnostics tool for detecting infectious or inflammatory foci. In view of the good performance of the Tc-99m-labeled PEG liposomes in detecting such foci, it is clear that radiolabeled liposomes could also be used as theranostic agents by loading them with antibiotics or anti-inflammatory drugs. This is particularly true for antibiotics because sub-optimal drug concentrations at the site of infection can lead to the appearance of bacterial resistance. Radiolabeled liposomes could help estimate the amount of antibiotic reaching the infectious foci and allow rapid adjustments of the therapeutic schedule.

\section{Respiratory Diseases}

An example of the aforementioned approach is given in a study by Weers et al. [25], using a Tc-99m-labeled liposomal formulation of the aminoglycoside amikacin, administered through a nebulizer. In this case, using aerosolized liposomes was intended to provide a slowrelease formulation, reducing dosing frequency, to increase drug penetration through the bacterial biofilm and to reduce systemic exposure to the drug. The study was performed in three healthy subjects and showed that nearly $40 \%$ of the deposited liposome dose was still present in the lungs $48 \mathrm{~h}$ after administration, indicating longer retention compared to non-encapsulated drugs. It also clearly showed the mucociliary escalator in action, with a large fraction of the deposited dose ending up in the stomach after being cleared upwards from the airways and swallowed. Although this radiolabeling approach could potentially have been useful for larger clinical trials, development of this liposomal antibiotic (Arikace ${ }^{\mathrm{TM}}$ ) appears to have halted in phase III. An earlier study of radiolabeled nebulized liposomes had also shown longer retention of the liposome-encapsulated tracer [26]. Bhavna et al. [27] compared the distribution of two Tc-99m-labeled, inhaled formulations of salbutamol (a long-acting $\beta 2$ agonist used in asthma and chronic obstructive pulmonary disease management) with different particle sizes. The hypothesis was that a reduced particle size would lead to improved drug delivery by increasing deposition in the peripheral lung alveoli and reducing uptake by alveolar macrophages. Although the latter aspect was not described in the study, scintigraphic images reveal deeper penetration of the smaller-sized formulation (average particle size $60 \mathrm{~nm}$ ) compared to the commercial formulation with a particle size of around $10 \mu \mathrm{m}$, as expected for inhaled particles [43]. Despite intense research efforts in nanomedicines for drug delivery to the lungs [44], very few clinical trials appear to have made use of the possibilities offered by non-invasive imaging techniques.

\section{Clinical Studies Using PET Imaging}

The most commonly used positron-emitting radionuclides in clinical studies are fluorine-18 $\left(\mathrm{F}-18, t_{1 / 2}=110 \mathrm{~min}\right)$, rubidium-82 $\left(\mathrm{Rb}-82, t_{1 / 2}=1.3 \mathrm{~min}\right)$, carbon-11 $(\mathrm{C}-11$, $\left.t_{1 / 2}=20.3 \mathrm{~min}\right)$, gallium-68 (Ga-68, $\left.t_{1 / 2}=67.8 \mathrm{~min}\right)$, copper-64 $\left(\mathrm{Cu}-64, t_{1 / 2}=12.7 \mathrm{~h}\right)$, and zirconium-89 (Zr-89, $t_{1 / 2}=3.27$ day). Positrons emitted from PET radioisotopes travel up to a short distance (positron range, up to a few $\mathrm{mm}$ ) before encountering an electron, at which point an annihilation event produces two $511-\mathrm{keV}$ gamma rays at a near $180^{\circ}$ angle. This pair of coincident gamma photons is detected by the PET camera, in which detectors are arranged in several static rings, providing 3D images. The coincidence detection allows to dispense with physical collimators used in SPECT/planar scintigraphy, thereby increasing the sensitivity of PET scanners by several orders of magnitude over SPECT scanners, in the range of $10^{-11}$ to $10^{-12} \mathrm{~mol} / \mathrm{l}$ of radiotracer, as well as the temporal resolution [35]. The lack of collimators also results in signals that are more easily quantifiable than those from gamma-emitting isotopes, and a maximum spatial resolution of approximately $5 \mathrm{~mm}$. Despite these advantages, only two recent clinical studies of nanomedicines using PET have had results published as of April 2018, both in the field of oncology.

In the context of a clinical trial (NCT01304797) of MM302, a formulation of PEGylated liposomal doxorubicin targeted against human epidermal growth factor receptor 2 (HER2), Lee et al. [28] selected 19 patients with metastatic breast cancer for an imaging study using MM-302 radiolabeled with $\mathrm{Cu}-64$. Current clinically approved formulations of PEGylated liposomal doxorubicin (Doxil $\AA /$ Caelyx ${ }^{\circledR}$, Myocet ${ }^{\circledR}$ ) do not possess targeting moieties and rely solely in the EPR effect. MM-302 is targeted towards HER-2 with the objective of increasing delivery of doxorubicin in HER2-overexpressing cells rather than in macrophages, as observed in a preclinical study [45].

The authors sought to determine whether the amount of drug reaching the metastases would correlate with 
therapeutic efficacy. Treatment with MM-302 was given along with trastuzumab (a clinically approved anti-HER2 monoclonal antibody). The chelating and loading agent 4DEAP-ATSC [46] was used to radiolabel MM-302 with $\mathrm{Cu}-$ 64 , with a target dose of $400 \mathrm{MBq}$ per patient. The maximum $\mathrm{Cu}-64$ activity remaining in the patients would be $108 \mathrm{MBq}$ after $24 \mathrm{~h}$ and $29 \mathrm{MBq}$ after $48 \mathrm{~h}$, potentially sufficient for PET imaging at this time point. Results from PET imaging (Fig. 3) showed that $\left[{ }^{64} \mathrm{Cu}\right] \mathrm{MM}-302$ remained in the circulation for over $24 \mathrm{~h}$, and thereafter accumulated mostly in the liver and spleen, in good agreement with preclinical data.

The authors state that free $\mathrm{Cu}-64$ was not detectable in the patients selected for more detailed analysis. This is not surprising, because free $\mathrm{Cu}-64$ has high affinity for liver and spleen tissues and rapidly accumulates in these organs [47]. Therefore, assessing the stability of the $\mathrm{Cu}-64$ radiolabeling of MM-302 based on PET images is not straightforward. Based on preclinical data showing that the biodistribution of MM-302 could be affected by treatment with cyclophosphamide, the patients selected for the imaging study were taken both from a group receiving MM-302 and trastuzumab and from a group receiving cyclophosphamide in addition. However, no differences in drug uptake were observed between these two groups, leading the patients to be analyzed as a single group for the rest of the study. There were several important results from the study. The first is the large heterogeneity in drug uptake not only between subjects but also between lesions within subjects. This high variability of the EPR effect is particularly important from a therapeutic point of view, since metastases exposed to insufficient drug concentrations could serve as starting points for further dissemination of cancer cells, potentially negating the initial benefits of the treatment. This led the authors to stratify the patients according to the amount of nanomedicine present in the lesion with the lowest uptake. Although the study was underpowered to show a correlation between MM-302 uptake and progression-free survival, an encouraging trend was observed that would warrant the enrollment of additional patients. A second lesson was the good agreement between clinical and preclinical data, both in overall nanomedicine distribution and in drug concentrations in the tumors. This should strengthen the case for clinical trials when imaging-based preclinical data are encouraging. Furthermore, the authors found no correlation between drug concentrations at the tumors and either drug concentrations in the blood or tumor size. This means that blood sampling will not be predictive of efficacy and highlights the added value of quantitative whole-body imaging techniques. A limitation of this study may paradoxically reside in the use of $\mathrm{Cu}-64$ as an imaging agent. PET imaging has also been used by several groups [48-50], to show that the maximal tumor uptake of PEGylated liposomes in preclinical models occurs within $24-48 \mathrm{~h}$ post-administration, but imaging at later time points (e.g., $72 \mathrm{~h}$ ) improves signal-to-background ratio. In practice, the half-life of $\mathrm{Cu}-64$ limits the imaging window to approximately $48 \mathrm{~h}$ post-administration and even this duration

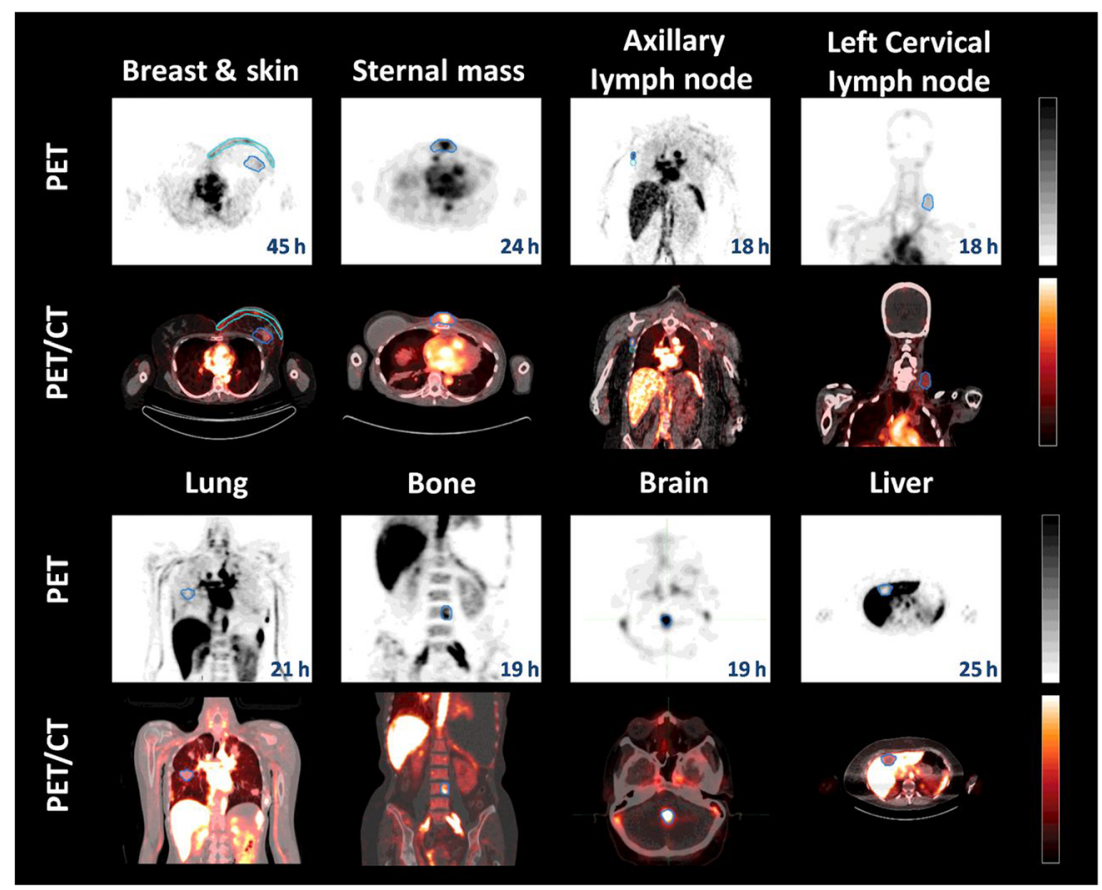

Fig. 3. Representative PET and fused PET/CT images of $\left[{ }^{64} \mathrm{Cu}\right]-\mathrm{MM}-302$ in lesions at different anatomic locations. The regions of interest used to measure tumor deposition of $\left[{ }^{64} \mathrm{Cu}\right]-\mathrm{MM}-302$ are shown in blue or turquoise outlines. Adapted with permission from the American Association for Cancer Research: Lee et al. [28]. 
requires high starting amounts of radioactivity. The use of longer-lived PET isotopes, such as $\mathrm{Zr}-89\left(t_{1 / 2}=3.27\right.$ day) or Mn-52 $\left(t_{1 / 2}=5.59\right.$ day $)$, should overcome this barrier.

The other clinical study of a PET-radiolabeled nanomedicine was conducted by Phillips et al. [29], using fluorescent nanoparticles conjugated to iodine-124 (I-124, $t_{1 / 2}=4.18$ day), for optical and PET imaging. The nanoparticles were also conjugated to an integrin-targeting peptide and engineered to promote renal clearance, and were therefore expected to have a distinct biodistribution pattern compared to liposomal nanomedicines. Indeed, the authors observed rapid clearance of the nanoparticles with most of the activity eliminated within $72 \mathrm{~h}$ and no accumulation in the liver and spleen. This is an interesting feature compared to liposomal nanomedicines, where liver and spleen accumulation complicates the visualization of nearby tumors. Although the study focused mainly on the safety and stability of the nanoparticles, accumulation of the nanoparticles was observed at tumor sites in some patients, with increasing target-to-background ratios over time but already observable within $4 \mathrm{~h}$ of administration. This favorable pharmacokinetic profile may enable faster clinical decisions. On the other hand, the nanoparticles were observed to accumulate in the renal cortices of a patient known to have chemotherapy-induced kidney inflammation, potentially complicating the differentiation between tumor areas and inflammatory lesions. There would be no reason to expect off-tumor inflammation in preclinical models of cancer, and therefore such a chance observation could only be made in a clinical study. This shows the value of incorporating whole-body imaging at the earliest opportunity in clinical studies of nanomedicines. Furthermore, the combination with an optical probe is an excellent choice for clinical applications of multimodal imaging, allowing tumor localization by PET to be followed by fluorescence-guided surgery for improved tumor resection, ultimately resulting in improved patient outcomes.

\section{Clinical Studies Using Magnetic Resonance Imaging}

In magnetic resonance imaging, the subject is placed in a powerful magnetic field, which will align magnetically active nuclei (most commonly hydrogen from water molecules) either parallel or anti-parallel to the magnetic field. The small difference in the number of nuclei aligning in each direction gives rise to the magnetic resonance imaging (MRI) signal. A pulsed radiofrequency can then be used to temporarily disturb the alignments of the nuclei, and the relaxation time back to the original position is measured. This relaxation time is dependent on the environment of the nuclei, for example, hydrogen nuclei in a fat-rich environment, or with short distance of an iron oxide nanoparticle, have shorter relaxation times than those in an aqueous environment. It is important to note that when using contrast agents in MRI, the measured signal arises not directly from the imaging agent but from the changes in magnetic properties the agent induces in its local environment. MRI has a much lower sensitivity than nuclear imaging techniques and requires imaging agent concentrations in the range of $10^{-3}$ to $10^{-5} \mathrm{~mol} / \mathrm{l}$, which can have pharmacological effects. Although MRI avoids the use of ionizing radiation, the inherent presence of MR-active nuclei means that at least two imaging sessions are necessary when imaging drug delivery: one before the administration of the MR-detectable agent, and one at a suitable time after administration. In addition, accurate quantification of the signal derived from contrast agents in MRI, particularly those based on superparamagnetic iron oxide materials, is difficult. On the other hand, the spatial resolution of clinical MRI is approximately $1 \mathrm{~mm}$, providing far more detailed images than PET or SPECT [35].

In a recent study, Ramanathan et al. [30] described an interesting approach to image-guided drug delivery. The aim was to use the tumor deposition of Ferumoxytol, a carboxydextran-coated superparamagnetic iron oxide nanoparticle with long circulation time and affinity for macrophages [51], as a surrogate marker for tumor deposition of nal-IRI (MM-398, Onivyde $($ ), a nanoliposomal formulation of the topoisomerase I inhibitor irinotecan. Both Ferumoxytol and nal-IRI are clinically approved products. The driving idea was that the amount of nanomedicine reaching the tumor would depend more on the vascular permeability at the tumor site and the average particle size than on the specific composition of the nanomedicine, so that iron nanoparticles and liposomes with otherwise comparable pharmacokinetics would reach the tumor in similar amounts. This study was performed in 13 patients with solid tumors, of which 9 were also assessed for treatment response by $\mathrm{CT}$. Ferumoxytol uptake was quantified by $\mathrm{T} 2 *$ imaging (Fig. 4), and irinotecan levels were measured from patient biopsies. There was no statistically significant correlation between Ferumoxytol uptake and irinotecan levels.

In a similar two-class approach to that used by Lee $e t$ al. [28] in the PET study of MM-302 described above, the authors classified lesions according to their Ferumoxytol uptake (below or above the median of uptake in all lesions). Using this approach, lesions with above-median Ferumoxytol uptake showed significantly improved changes in size after nal-IRI treatment over those with below-median uptake. Another major finding of this study was the high variability of Ferumoxytol between patients and between lesions in a given patient, echoing the high variability of the EPR effect reported for Tc-99m- and Cu-64-labeled PEGylated liposomes $[19,28]$. This MRI-based study takes a "companion diagnostic" approach akin to that used in the TSC/Doxil ${ }^{\circledR}$ combination study by Giovinazzo et al. [23]. Since the uptake of one drug is used as an indirect reflection of the uptake of a second therapeutic drug, there is an inherent possibility of pharmacokinetic/biodistribution differences between the two drugs. Correlations must therefore be established in preliminary studies for each envisaged reporter/drug combination before the pair can be used in the clinic. However, the use of clinically approved imaging agents could easily be integrated into existing treatment 

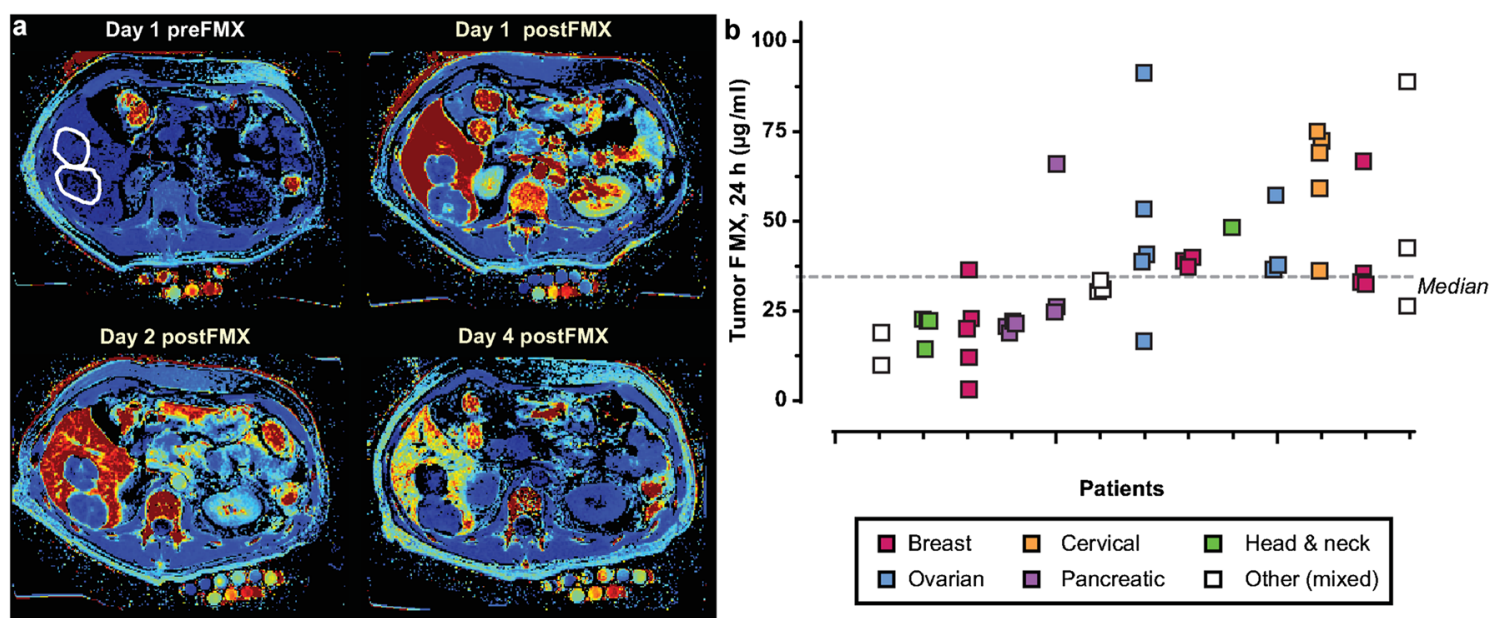

Fig. 4. a Representative pseudocolored relaxometric R2* maps derived from patient images before and after administration of iron oxide nanoparticle (Ferumoxytol $尺, F M X)$. Approximate lesion locations are indicated by white lines in the image before FMX dosing. b Extrapolated FMX concentrations in individual patients $24 \mathrm{~h}$ after administration. Adapted with permission from the American Association for Cancer Research: Ramanathan et al. [30].

protocols by not having to go through the same regulatory hurdles as theranostic agents, which could be considered as new drugs and therefore require full regulatory approval.

Two other clinical trials (NCT02022644 and NCT03086616) of nal-IRI that include image-guided drug delivery are planned. Convection-enhanced delivery (CED) of nal-IRI to the brain of pediatric and adult patients with glioma will be followed in real time by MRI, using co-administered gadolinium-based contrast agents.

\section{Clinical Studies Using Ultrasound Imaging}

Ultrasound imaging relies on sending sound waves through the body and recording the reflected waves to produce a high-resolution 2D image. This is done with a hand-held probe, making it a widely available and very cost-effective technique, but is mostly limited to soft tissue imaging [35]. There is a large body of preclinical work on focused ultrasound (FUS)-mediated drug delivery, especially using thermosensitive liposomes, with the imaging aspect typically performed by ultrasound or MRI. For further information on this topic, the reader is directed to recent and extensive reviews [52-54]. We have found only one report related to ultrasound-guided drug delivery in humans. Lyon et al. [31] recently described the protocol of an ongoing (as of April 2018) clinical trial of ThermoDox ${ }^{\circledR}$, a thermosensitive liposomal formulation of doxorubicin with FDA approval for investigational use. By using low-intensity FUS to visualize liver tumors and induce mild hyperthermia, the aim is to selectively increase the concentration of doxorubicin at the tumor sites. Doxorubicin levels will be measured by high-performance liquid chromatography in biopsied tumor tissues before and after application of FUS. Only the first few patients are to undergo tumor biopsy before FUS, to establish an average tumor concentration of doxorubicin prior to FUS application. The authors describe this as a way of reducing the number of invasive procedures performed on the patients. However, considering the variability of liposomal uptake in tumors highlighted in the MM-302 study [28], we caution that this approach might yield misleading results. It would be preferable, especially for a pilot study, to determine pre-FUS doxorubicin concentration in as many patients as possible to adequately quantify the effect of FUS on liposomal drug release. Other imaging modalities such as $\left[{ }^{18}\right.$ F]FDG PET-CT and dynamic-contrast enhanced (DCE)MRI are included in the study, but aimed at establishing baseline tumor imaging and potential response to treatment rather than evaluating drug delivery. Results from this study have yet to be published; however, it will be of considerable interest to see whether a non-invasive and non-ionizing method such as FUS can be used to increase doxorubicin concentrations above therapeutic threshold specifically in cancerous tissue to minimize the effect on healthy tissues.

\section{Conclusions and Perspectives}

Imaging has been successfully used in humans to study the biodistribution and pharmacokinetics of nanomedicine-based drug delivery systems, demonstrating its value for this purpose. Significant findings from these studies include solid proof that (1) EPR as the most common uptake mechanism for nanomedicines in tumors/inflamed tissues is a real phenomenon in patients and (2) the EPR effect is highly heterogeneous, between diseases, patients, and even lesions within a single patient. This heterogeneity may underlie the fact that nanomedicines have not always shown superiority in clinical therapeutic activity over conventional drugs, despite an improved safety profile [11]. Indeed, an absence of therapeutic effect does not imply absence of 
nanomedicine accumulation in tumors. This is particularly relevant for metastatic cancers, as nanomedicine accumulation only at certain tumor sites may not be sufficient for an overall survival benefit to the patient. Moreover, recent clinical studies have demonstrated a clear correlation between nanomedicine accumulation levels in target tissues (e.g., tumors) and therapeutic effect, supporting the use of imaging to identify patients that will respond to the treatment. An important concept that arises from these studies is the development of companion diagnostics, based on clinically approved imaging agents that correlate with nanomedicine biodistribution. Another conclusion from this review is that most of the studies to date have been in the oncology field, leaving further opportunities for imageguided drug delivery studies beyond this area, particularly in the fields of infection and respiratory medicine.

A general limitation of the nanomedicine-imaging approaches described in this review is that they are in practice indirect methods. Indeed, the imaging agent is most frequently attached to the carrier rather than to the drug, whereas clinically the most relevant information is the localization of the drug itself, which may differ from that of the carrier after release. This would generally require chemical modification of the drug. Furthermore, it is extremely challenging to differentiate between nanocarrier-bound and released drug by non-invasive imaging. This may be possible by optical imaging, especially for intrinsically fluorescent drugs, in vitro and possibly at the preclinical level, but not in humans [55]. An option is coloading liposomes with a drug and a magnetic resonance (MR) contrast agent, as the relaxivity of the encapsulated and nonencapsulated MR contrast agent will differ [56], but this method suffers from the inherent limitations of MRI discussed above (sensitivity, challenging whole-body detection/quantification). Furthermore, it will not inform on drug distribution after release and at later times. Nuclear imaging modalities may be helpful in this context, for example, by loading radiolabeled drugs into nanocarriers; however, the half-lives of the radioisotopes most amenable to radiolabeling of small molecules (e.g., C-11 and F-18) are too short to match the biological half-lives of nanomedicines. In this context, a recent preclinical study demonstrated the concept of radiolabeling both the liposome carrier and the encapsulated drug with PET radionuclides [57]. Another possibility is to use multi-isotope SPECT and image both the carrier and the drug orthogonally; however, this is only possible when using radionuclides with different gamma emission energies (e.g., Tc-99m and In-111). Longer imaging windows are attainable by using metal-chelating drugs and radiometals such as $\mathrm{Zr}-89$ [48]; however, this raises another issue, which is the stability of the free radionuclide/ label. Even the longer-lived and covalently bound radioiodine (I-124, I-125, I-131) labels are susceptible to deiodination in vivo. As mentioned previously, the accumulation of the free radiolabel in specific anatomical locations (e.g., the thyroid for radioiodine, bones for $\mathrm{Zr}-89$, or pancreas for Mn-52 [58]) may inform on the extent of drug release from the carrier. Even this requires the assumption that the rate of release of the radiolabel from the drug is similar to the drug release from the carrier and will not inform about drug localization.

Despite all these issues, it is clear that among the different imaging techniques available, nuclear medicine techniques have been the most used to date, most likely due to their wholebody capabilities and excellent quantification properties. Importantly, their high sensitivity also allows imaging with sub-therapeutic nanomedicine doses in the microdose range ( $1 \%$ of the therapeutic dose), which facilitates clinical translation. MRI and US circumvent the use of radioactivity and provide higher spatio-temporal resolution, at the expense of lower sensitivity and higher imaging agent doses. In addition, performing quantitative whole-body imaging with MRI and US is a more complex process than with nuclear imaging. For these reasons, it is likely that future clinical studies in this area will include nuclear imaging as the main quantitative method to assess nanomedicine/drug concentration in vivo. The recent study by Lee et al. [28] using $\left[{ }^{64} \mathrm{Cu}\right] \mathrm{MM}-302$ is an excellent example of the way clinical image-guided drug delivery studies should be conducted nowadays, particularly for its approach to patient stratification. We believe that incorporating imaging into studies of nanomedicine drug delivery will undoubtedly provide valuable additional information that standard techniques such as blood sampling and biopsy cannot provide. This will greatly benefit the clinical development of new nanomedicines and help achieve the full potential of those already developed.

Acknowledgments. We thank the European Society for Molecular Imaging (ESMI) for their support and the possibility of establishing a study group for Image-Guided Drug Delivery (IGDD) as a platform for scientific exchange within the society and beyond. F. M. and R.T.M.R are funded by a CRUK Multidisciplinary Project Award [grant number C48390/A21153], the KCL/ UCL Comprehensive Cancer Imaging Centre funded by CRUK and EPSRC in association with the MRC and DoH (England), the Wellcome EPSRC Centre for Medical Engineering at KCL [grant number WT 203148/Z/16/Z], the Medical Research Council Confidence in Concepts scheme, the Experimental Cancer Medicine Centre at KCL, the KHP/KCL CRUK Cancer Centre and the National Institute for Health Research (NIHR) Biomedical Research Centre based at Guy's and St Thomas' NHS Foundation Trust and KCL [grant number IS-BRC-1215-20006]. The views expressed are those of the authors and not necessarily those of the NHS, the NIHR or the Department of Health. T.L. gratefully acknowledges support by the European Research Council (ERC starting grant NeoNaNo (309495) and ERC Proof-of-Concept grants CONQUEST (680882) and PIcelles (813086)) and the German Research Foundation (DFG; La2937/1-2, SFB1066, GRK2375).

\section{Compliance with Ethical Standards}

\section{Ethical Approval}

This article does not contain any studies with human participants or animals performed by any of the authors.

\section{Conflict of Interest}

The authors declare that they have no conflict of interest.

Open Access This article is distributed under the terms of the Creative Commons Attribution 4.0 International License (http:// creativecommons.org/licenses/by/4.0/), which permits unrestricted use, distribution, and reproduction in any medium, provided you give appropriate credit to the original author(s) and the source, provide a link to the Creative Commons license, and indicate if changes were made. 


\section{References}

1. Farokhzad OC, Langer R (2009) Impact of nanotechnology on drug delivery. ACS Nano 3:16-20

2. Kunjachan S, Ehling J, Storm G et al (2015) Noninvasive imaging of nanomedicines and nanotheranostics: principles, progress, and prospects. Chem Rev 115:10907-10937

3. Chakravarty R, Hong H, Cai W (2014) Positron emission tomography image-guided drug delivery: current status and future perspectives. Mol Pharm 11:3777-3797

4. Ojha T, Rizzo L, Storm G et al (2015) Image-guided drug delivery: preclinical applications and clinical translation. Expert Opin Drug Deliv 12:1203-1207

5. Lammers T, Kiessling F, Hennink WE, Storm G (2010) Nanotheranostics and image-guided drug delivery: current concepts and future directions. Mol Pharm 7:1899-1912

6. Lanza GM, Moonen C, Baker JR et al (2014) Assessing the barriers to image-guided drug delivery. Wiley Interdiscip Rev Nanomedicine Nanobiotechnology 6:1-14

7. Chakravarty R, Hong H, Cai W (2015) Image-guided drug delivery with single-photon emission computed tomography: a review of literature. Curr Drug Targets 16:592-609

8. Prabhakar U, Maeda H, Jain RK et al (2013) Challenges and key considerations of the enhanced permeability and retention effect for nanomedicine drug delivery in oncology. Cancer Res 73:2412-2417

9. Lammers T, Rizzo LY, Storm G, Kiessling F (2012) Personalized nanomedicine. Clin Cancer Res 18:4889-4894

10. Natfji AA, Ravishankar D, Osborn HMI, Greco F (2017) Parameters affecting the enhanced permeability and retention effect: the need for patient selection. J Pharm Sci 106:3179-3187

11. Hare JI, Lammers T, Ashford MB et al (2017) Challenges and strategies in anti-cancer nanomedicine development: an industry perspective. Adv Drug Deliv Rev 108:25-38

12. Lopez-Berestein G, Kasi L, Rosenblum MG et al (1984) Clinical pharmacology of ${ }^{99 \mathrm{~m}} \mathrm{Tc}$-labeled liposomes in patients with cancer. Cancer Res 44:375-378

13. Turner AF, Presant CA, Proffitt RT et al (1988) In-111-labeled liposomes: dosimetry and tumor depiction. Radiology 166:761-765

14. Presant CA, Proffitt RT, Turner AF et al (1988) Successful imaging of human cancer with indium-111-labeled phospholipid vesicles. Cancer 62:905-911

15. Presant CA, Ksionski G, Crossley R (1990) ${ }^{111}$ In-labeled liposomes for tumor imaging: clinical results of the International Liposome Imaging Study. J Liposome Res 1:431-436

16. Presant CA, Blayney D, Kennedy P et al (1990) Preliminary report: imaging of Kaposi sarcoma and lymphoma in AIDS with indium-111labelled liposomes. Lancet 335:1307-1309

17. Khalifa A, Dodds D, Rampling R et al (1997) Liposomal distribution in malignant glioma: possibilities for therapy. Nucl Med Commun $18: 17-23$

18. Stewart S, Harrington KJ (1997) The biodistribution and pharmacokinetics of stealth liposomes in patients with solid tumors. Oncology 11:33-37

19. Harrington KJ, Mohammadtaghi S, Uster PS et al (2001) Effective targeting of solid tumors in patients with locally advanced cancers by radiolabeled pegylated liposomes effective targeting of solid tumors in patients with locally advanced cancers by radiolabeled pegylated liposomes. Clin Cancer Res 7:243-254

20. Koukourakis MI, Koukouraki S, Giatromanolaki A et al (1999) Liposomal doxorubicin and conventionally fractionated radiotherapy in the treatment of locally advanced non-small-cell lung cancer and head and neck cancer. J Clin Oncol 17:3512-3521

21. Koukourakis MI, Koukouraki S, Giatromanolaki A et al (2000) High intratumoral accumulation of stealth liposomal doxorubicin in sarcomas: rationale for combination with radiotherapy. Acta Oncol (Madr) 39:207-211

22. Murray JL, Kleinerman ES, Cunningham JE et al (1989) Phase I trial of liposomal muramyl tripeptide phosphatidylethanolamine in cancer patients. J Clin Oncol 7:1915-1925

23. Giovinazzo H, Kumar P, Sheikh A et al (2016) Technetium Tc $99 \mathrm{~m}$ sulfur colloid phenotypic probe for the pharmacokinetics and pharmacodynamics of PEGylated liposomal doxorubicin in women with ovarian cancer. Cancer Chemother Pharmacol 77:565-573
24. Dams ET, Oyen WJ, Boerman OC et al (2000) ${ }^{99 \mathrm{~m}}$ Tc-PEG liposomes for the scintigraphic detection of infection and inflammation: clinical evaluation. J Nucl Med 41:622-630

25. Weers J, Metzheiser B, Taylor G et al (2009) A gamma scintigraphy study to investigate lung deposition and clearance of inhaled amikacin-loaded liposomes in healthy male volunteers. J Aerosol Med Pulm Drug Deliv 22:131-138

26. Farr S, Kellaway I, Parry-Jones D, Woolfrey S (1985) 99mTechnetium as a marker of liposomal deposition and clearance in the human lung. Int J Pharm 26:303-316

27. Bhavna AFJ, Mittal G et al (2009) Nano-salbutamol dry powder inhalation: a new approach for treating broncho-constrictive conditions. Eur J Pharm Biopharm 71:282-291

28. Lee H, Shields AF, Siegel BA et al (2017) ${ }^{64} \mathrm{Cu}-\mathrm{MM}-302$ positron emission tomography quantifies variability of enhanced permeability and retention of nanoparticles in relation to treatment response in patients with metastatic breast cancer. Clin Cancer Res 23:41904202

29. Phillips E, Penate-Medina O, Zanzonico PB et al (2014) Clinical translation of an ultrasmall inorganic optical-PET imaging nanoparticle probe. Sci Transl Med 6:260ra149-260ra149

30. Ramanathan RK, Korn RL, Raghunand N et al (2017) Correlation between ferumoxytol uptake in tumor lesions by MRI and response to nanoliposomal irinotecan in patients with advanced solid tumors: a pilot study. Clin Cancer Res 23:3638-3648

31. Lyon PC, Griffiths LF, Lee J et al (2017) Clinical trial protocol for TARDOX: a phase I study to investigate the feasibility of targeted release of lyso-thermosensitive liposomal doxorubicin (ThermoDox $\left.{ }^{\circledR}\right)$ using focused ultrasound in patients with liver tumours. J Ther Ultrasound 5:28

32. Moek KL, Giesen D, Kok IC et al (2017) Theranostics using antibodies and antibody-related therapeutics. J Nucl Med 58:83S-90S

33. Petersen AL, Hansen AE, Gabizon A, Andresen TL (2012) Liposome imaging agents in personalized medicine. Adv Drug Deliv Rev 64:1417-1435

34. Lamichhane N, Udayakumar T, D'Souza W et al (2018) Liposomes: clinical applications and potential for image-guided drug delivery. Molecules 23:288

35. James ML, Gambhir SS (2012) A molecular imaging primer: modalities, imaging agents, and applications. Physiol Rev 92:897-965

36. Gregoriadis G, Swain CP, Wills EJ, Tavill AS (1974) Drug-carrier potential of liposomes in cancer chemotherapy. Lancet 303:1313-1316

37. Segal AW, Gregoriadis G, Lavender JP et al (1976) Tissue and hepatic subcellular distribution of liposomes containing bleomycin after intravenous administration to patients with neoplasms. Clin Sci $51: 421-425$

38. Richardson VJ, Ryman BE, Jewkes RF et al (1979) Tissue distribution and tumour localization of $99 \mathrm{~m}$-technetium-labelled liposomes in cancer patients. Br J Cancer 40:35-43

39. Perez-Soler R, Lopez-Berestein G, Kasi LP et al (1985) Distribution of technetium-99m-labeled multilamellar liposomes in patients with Hodgkin's disease. J Nucl Med 26:743-749

40. Immordino ML, Dosio F, Cattel L (2006) Stealth liposomes: review of the basic science, rationale, and clinical applications, existing and potential. Int J Nanomedicine 1:297-315

41. Gabizon A, Catane R, Uziely B et al (1994) Prolonged circulation time and enhanced accumulation in malignant exudates of doxorubicin encapsulated in polyethylene-glycol coated liposomes. Cancer Res 54:987-992

42. Thakor AS, Jokerst JV, Ghanouni P et al (2016) Clinically approved nanoparticle imaging agents. J Nucl Med 57:1833-1837

43. Heyder J (2004) Deposition of inhaled particles in the human respiratory tract and consequences for regional targeting in respiratory drug delivery. Proc Am Thorac Soc 1:315-320

44. van Rijt SH, Bein T, Meiners S (2014) Medical nanoparticles for next generation drug delivery to the lungs. Eur Respir J 44:765-774

45. Kirpotin DB, Drummond DC, Shao Y et al (2006) Antibody targeting of long-circulating lipidic nanoparticles does not increase tumor localization but does increase internalization in animal models. Cancer Res 66:6732-6740

46. Lee H, Zheng J, Gaddy D et al (2015) A gradient-loadable ${ }^{64} \mathrm{Cu}-$ chelator for quantifying tumor deposition kinetics of nanoliposomal therapeutics by positron emission tomography. Nanomedicine $11: 155-165$ 
47. Avila-Rodriguez MA, Rios C, Carrasco-Hernandez J et al (2017) Biodistribution and radiation dosimetry of $\left[{ }^{64} \mathrm{Cu}\right]$ copper dichloride: first-in-human study in healthy volunteers. EJNMMI Res 7:98

48. Edmonds S, Volpe A, Shmeeda H et al (2016) Exploiting the metalchelating properties of the drug cargo for in vivo positron emission tomography imaging of liposomal nanomedicines. ACS Nano 10:10294-10307

49. Ngoune R, Peters A, von Elverfeldt D et al (2016) Accumulating nanoparticles by EPR: a route of no return. J Control Release 238:58-70

50. Pérez-Medina C, Abdel-Atti D, Tang J et al (2016) Nanoreporter PET predicts the efficacy of anti-cancer nanotherapy. Nat Commun 7:11838

51. Daldrup-Link HE, Golovko D, Ruffell B et al (2011) MRI of tumorassociated macrophages with clinically applicable iron oxide nanoparticles. Clin Cancer Res 17:5695-5704

52. Hijnen N, Langereis S, Grüll H (2014) Magnetic resonance guided high-intensity focused ultrasound for image-guided temperatureinduced drug delivery. Adv Drug Deliv Rev 72:65-81
53. Wright M, Centelles M, Gedroyc W, Thanou M (2018) Image guided focused ultrasound as a new method of targeted drug delivery. In: Theranostics and image guided drug delivery. Ed.Thanou M. London: The Royal Society of Chemistry, pp 1-28

54. Kiessling F, Fokong S, Bzyl J et al (2014) Recent advances in molecular, multimodal and theranostic ultrasound imaging. Adv Drug Deliv Rev 72:15-27

55. Etrych T, Lucas H, Janoušková O et al (2016) Fluorescence optical imaging in anticancer drug delivery. J Control Release 226:168-181

56. Negussie AH, Yarmolenko PS, Partanen A et al (2011) Formulation and characterisation of magnetic resonance imageable thermally sensitive liposomes for use with magnetic resonance-guided high intensity focused ultrasound. Int J Hyperth 27:140-155

57. Lamichhane N, Dewkar GK, Sundaresan G et al (2017) [18F]Fluorinated carboplatin and [111In]-liposome for image-guided drug delivery. Int J Mol Sci 18:1079

58. Gawne P, Man F, Fonslet J, et al. (2018) Manganese-52: applications in cell radiolabelling and liposomal nanomedicine PET imaging using oxine (8-hydroxyquinoline) as an ionophore. Dalt Trans 47:92839293 